\title{
La salud mental de base un campo con mayor
responsabilidad cada dia
}

"Extomas viviendo un momento en que se tiende a complejizar permanentemente la explicación de las hechos Se producen andisis complicadisimas - desirididas a grupas selectos-sobre simaciones simples porgine la complicación este al servicio de la confisidn y tria, a su ver, es un arma de dominio".

Franco Basaglia.

La salud mental de base es un campo nuevo y, por eso mismo, no muy claramente reconocido. Cuasio los bancos internacionales y similares (AID, Banco Mundial, Fondo Monetario Internnclonal, Banco Interamericano de Desarrollo) decidieron hacer verdadens invertioses en materia de salud, alli por los afos ochenta, surgio sin mayor preocupactch institucional, o mús bien como "maquillaje modernizante", el campo de la aresción primaria en salud mental. Entonces se entendió que era llevar ealud mentel a les pobleciones carentes de todo. Donde conseculentemente no exietinn ervicios especializados.

Ya admitida la necesided de una metodología propia, basada en el trabajo comunitario, la propuesta inicial adquirió visibilidad y denominación (salud mental de base) por el lado de "contener" crisis colectivas, a veces masivas, en poblaciones sometidas a situaciones de "máximo riesgo". Es decir, gente que compartía una condición humanamente tulgica, pero no tanto como producto de un desastre eventual y efímero, sino mís propiamente como una vivencis inevitable y prolongada (pobreza, guerrilla, destrucción medioambiental no tecnificada).

En Centroamerica no nos llevo demasiado tiempo comprender que cuando trabajabamos en salud mental de bese, nos enfrentábamos fundacionalmente a algo más profundo y transanitario que la carencia de víveres, la tala de ́́rboles y los 
asesinados. Algo que estaba a la base de todos esos fenómenos que afectaban la salud mental. Parecía poco técnico decirlo, por su incidencia política, pero medularmente nos estábamos enfrentando a las consecuencias, en la salud mental, de la violencia estructural (de una patogénesis institucionalizada, o de una lógica sistémica enfermiza).

A pesar de la orfandad térico-conceptual, comprendimos técnicamente la importancia de trabajar sobre siruaciones antes que sobre patologlas o individuos. Así no sólo pudimos ubicar la afectación (o enfermedad) en las relaciones (nivel relacional) entre los grupos y las personas (fuera del individuo), también le agregamos el efecto de las instituciones (formales e infornales) sobre la salud mental de base de cada ciudadano. Esto fue posible gracias a la vision de la forma en que la vida cotidiana codifica la subjetividad personal, y la determinación de esta cotidianeidad por parte de las instiluciones de la sociedad (visión aportada complementariamente por Martin-Baró y Franco Basaglia).

Poco a poco se hizo evidente de que, si bien encontrábamos situaciones espectficas de afectación que daban lugar a síntomas también especificos (situaciones psicosomatógenas, obsesivógenas, esquizofrenizantes, etc.), que más propiamente podrlamos llamar microsituaciones, habla un cúmulo de manifestaciones clínicamente inespecificas, asociadas a condiciones psicosociales más elementales, las cuales, además, parecen servir de base para el desarrollo de las situaciones especificas

El paso siguiente fue identificar cuál era el tipo de esas condiciones psicosociales más elementales. Inicialmente hablábamos de la insatisfacción de las necesidades humanas básicas (que era evidente en las comunidades atendidas en la fpoca de la guerra). Pensábamos que esas necesidades humanas, ostensiblemẹpte insatisfechas, generaban los sintomas variables e inespecfficos encontrados a través de un proceso psicosocial prolongado de "ambientación" de la impotencja (que fue llamado por Luciano Carrino: ciclo afectivo negativo).

Se identifica un síntoma poco dramático, pero muy "de fondo" y constante, caracterizado por: insatisfacción con sf mismo, displacer, pédida dol apasionamiento por involucrarse en planes de cambio y disminución del interess por prever las nuevas secuencias posibles de la "película de la vida". Sin embargo, profundizando en el estudio de las siluaciones de insatisfacción de las necesjdedes humanas básicas, tuvimos que admitir que dentro de ellas habla un elemento invisible pero más esencial y determinante, que no era en sf misma la falta de cobertura a las necesidades identificadas. Asl, para la salud mental de base no era lo mismo el hambre de un guerrillero ejercida voluntariamente (como sujeto), que el hambre de un refugiado, asumida pasiva e independientemente de la voluntad (como la lluvia, la noche y las estaciones, sólo que mucho más psicolesivamente por su carácter interhumano y restrictivo del ejercicio de poder socio-relacional de la persona). 
Cuando contrastamos las diferentes consecuencias en la salud mental de base de le misna hambre física (y demós necesidades básicas insatiffechas), es decir, de la soportada por decisión propia y de la "caida del cielo", comprobamoe que existía un factor cualitativo: el sentido que tenía el hecho para cada persona. Pars el guerrillero, esa hambre reprosentabs una cansagración de su dignidad e identidad, y un paso en la conquista de un ideal. Poseía, por lo tanto, pleno sentido. En el caso opueato, para la vectima pasiva de la guerra, el hambre era solo un sufrimiento sin sentido ante el cual no podia hacer nada. Ahora, alcanzamos el punto de precisar: ¿Qué entendemos cuando hablamos de que la situsción tiene o no sentido? Calificamos no por un juicio de valor que la situación tenga sentido (el hambre del guerrillero), sino porque le permita a la persona funcionar como sujew. Si la situación (interhumana) no le permite al individuo erigirse como sujeto, de alguna forma (ya sea produciendo el cambio o sacándole provecho a la situsción) no tendra sentido pari a. Al funcionar como sujeto, la persona ejerce su poder socio-relacional. Definimos a este, siguiendo a Martín-Baro, corno la capacidad de satisfacer las propias necesidades humanas por medio de las relaciones sociales.

Legamos a la conclusión de que el determinante fundamental para que una situación no-especifica pueds coneiderarse afectante, es la falta de ejercicio de poder socia-relacional. Lo que afuctab la salud mental de base en los refugios y comunidades perjudicades por la guem no ere simplemente la insatisfacción de las necesidades humanas básicas, sino esa insatisfacción en cuanto a la privación del ejercicio de poder socio-relacional.

Muchos expertos en la atencion do poblaciones, en situaciones de graves privaciones, establecieron el tormbno resillencia para definir una resistencia superior para soportar los sufrimientos de h vida. En realidad, planteada en una forma mecanicista, la palabre parecerfe aludir a un poder mistico, y no ayudaría a investigar el proceso por el cual al identificado "resiliente" consigue erigirse como sujeto de la situación aparantormente muy adversa.

Fuera de las situeciones fracamente forzadas de la guerra, hay un indicador objetivo que nos permite detar el grado de privaciones de ejercicio de poder socio-relacional de las situsciones: la inequidad de ingresos. Un siglo atrás, las necesidades de los trabajedores eran sólo de techo, comida y ropa sencillos; sin luz, ni agua potable, ni televisión, ni revistas, ni teléfono, ni automóvil, ni vacaciones (el descanso semanal era de medio dia, los domingos y con obligación de ir a misa). Pero no sufrían de epidemias de afectaciones de la salud mental de base. En cambio, si sometiéramos a un grupo de trabajadores de una ciudad del "primer mundo" a esas condiciones de vida, príctimanente todos presentarlan alguna afectación de su salud mental de base. En una comprobación vivida real y lotalmente en nuestro tiempo, se ha demostrado muchas veces que las manifestaciones de violencia, entre las personas de una población determinada, no siguen fielmente al 
descenso o ascenso de las condiciones materiales (monto de los salarios), sino de lns variaciones de equidad. Es decir, del aumento o de la disminución relativas de la diferencias a nivel adquisitivo (diferencias salariales). Cuanto més diferencias de ingresos, mes violencia ¿Qué quiere decir esto pare la salud mental de base? Primero, que lo que se esth midiendo a traves de la variación de diferencia de ingreso como indicador, que podrlamos llamar variacion de la equidad, es el ejercicio de poder socio-relacional de los integrantes de esa población estudiada. Segundo, que la disminución del ejercicio de poder socio-relacional es lo que afecta la calud mental de base.

¿Por qué seńalamos a la equidad-inequidad como indicador primordial de la falta de ejercicio de poder socio-relacional? Bueno, porque el poder socio-rebacional se usa, precisamente, para satisfacer las propias necesidades humanas; hacia eso se dirige. (No hablemos de poder físico, sino de la capacidad de satisfacer las propias necesidades humanas a través de las relaciones saciales). Entonces, en el caso del trabajador del siglo pasado, no sufría por la falta de un televisor, etc, porgue aún no se le habla creado esa necesidad; no ejercía su poder socio-relacional para poseer televisor y, por lo tanto, la falta de un televisor no significaba limitación del ejercicio de poder socio-relacional; ni obviamente inequidad en relactón con el resto de la población que tampoco lo poseía. Pero, en un obrero suizo actual, el no tener un vehlculo es vivido como inequidad. De manera que si no accede a su automóvil es porque tiene una falta de ejercicio de poder socio-relacional.

De donde: $\Delta$ inequidad $=\Delta$ ejercicio de poder socio-relacional.

Un mayor ingreso o la posesión de mayores bienes y capacidades brinda a una persona mayor poder transaccional (en un sentido psicosocial y cotidinno) frente a los dernás. (Absirayéndolo del aspecto no-interactivo que olorgue el usuiructo de recursos materiales que mul(ipliquen la capacidad personal).

El otro componente del poder socio-relacional es el ecceso a redes saciales, 10 cual se pone dramáticamente en evidencia en Centroarnérica cuando se comprueba que muchas familias consiguen menores ingresos que el valor de la alimentación de todos sus miembros y, sin embargo, mueren de hambre. Las múltiples y pequeñas transacciones cotidianas logradas medianle la activación de sus redes cociales los mantienen vivos. Cuanto mayor acceso a redes sociales tiene una persona: mayor es su poder socio-relacional. (Claro que las redes se ubican, a su vez, en distintos niveles de poder material.)

Y aquí llegamos a una conclusión matemáticamente slamante para el futuro mundo globalizado y regido por la culrura urbano-informático-consumista: si el mantenimiento de la salud mental de base implica el ejercicio del poder sociorelecional y Este se consigue haciendo pesar los ingresos-bienes-capacidades y/o accediendo a redes sociales, al disminuir las redes sociales (que es la lendencia actuaf), la gente necesilará cada vez mayores ingresos económicos para mantener 
su salud mental de base. Es decir, al absolutizar la globalización el criterio de eficiencis, se ha inicisdo un proceso progresivo de deterioro de las redes sociales que hace caer el ejercicio del poder socio-relacional y (en consecunencin) el mantenimiento de he salud mental de base, progresivamente, en el nivel de ingresosbienes-capacided.

Pero no se necesita de una guerra para encontrar situaciones que priven a las personas del ejercicio de poder socio-relacional. En realidad, la misión de la salud mental de base es el estudio y atención de estas situaciones a las cuales denominamos: siruación primaria. Pera destacar su naturaleza elemental, la situación primaria puede ser producto de le propia lógica de funcionarniento del macrosistema social descalificante-exchyento o, en casos especiales, consecuencia de configuraciones relacionales particulare.

El resultado de la simincion primaria es lo que llamamos "afectación de base". Esta se desarrolla a trevits de siete procesos psicosociales, en el siguiente orden correlativo ("en cascact"): L debilitacion de los ámbitas psicasociales coridianos o sistemas cointegradores de la subjetividad personal la perdida de rales, la debilitación de la idenitdad la inmovilidad afectiva, la pérdida de conciencia social operativa, la incapacidat de planificación estratégica personal y, consecuentemente, una manifestación de violencia irracional. (Esta última no se opone al sistema generador sino que, dentro de ciertos límites de control, lo favorecen al acelerar la descalificacion vertical es decir, es útil al sistema.)

La situación primaria se tracuce umbién a nivel clínico (como, trastomos psicosomáticos del sueño, de le concentración, de la memoria, del aprendizaje, cansancio, irritabilidad, impulsivided, majestrr indefinido, adicciones (alcoholismo). A nivel psicosocial genera violencin intrafarniliar y consumismo compulsivo. Pero, además, deteriora gravemente el fuctor fundamental de prolección de la salud mental, como es la movilizucion afuctive a traves de las relaciones prosociales y de solidaridad humana. Dofinimos alud mental en terminos de la actitud más integral a desarrollar y no como un ettado, mectinicamente concebido, que aparece y desaparece por "arte" de uan norma institucional e ideológica.

Cada día, con mayor clarided, se comprende la necesidad de desarrollar suficientemente una rama interdisciplinaria de la investigación que, apoyándose en la psicología social, las ciencias politicas y la antropologia, tome como amplio objeto de estudio la influencia de las instituciones sobre la salud mental de base de la población. Entendida la salud mental de base como: el bienestar integral derivado de la satisfacción de las propias necesidades hwmanas básicas por medio de las relaciones sociales.

Creemos que estamos ante la responsabilidad de estudiar las consecuencias para la salud mental de base de las relaciones de poder, de descalificación y anulación de la capacidad contractual que son vitales a muchos sistemas más allá 


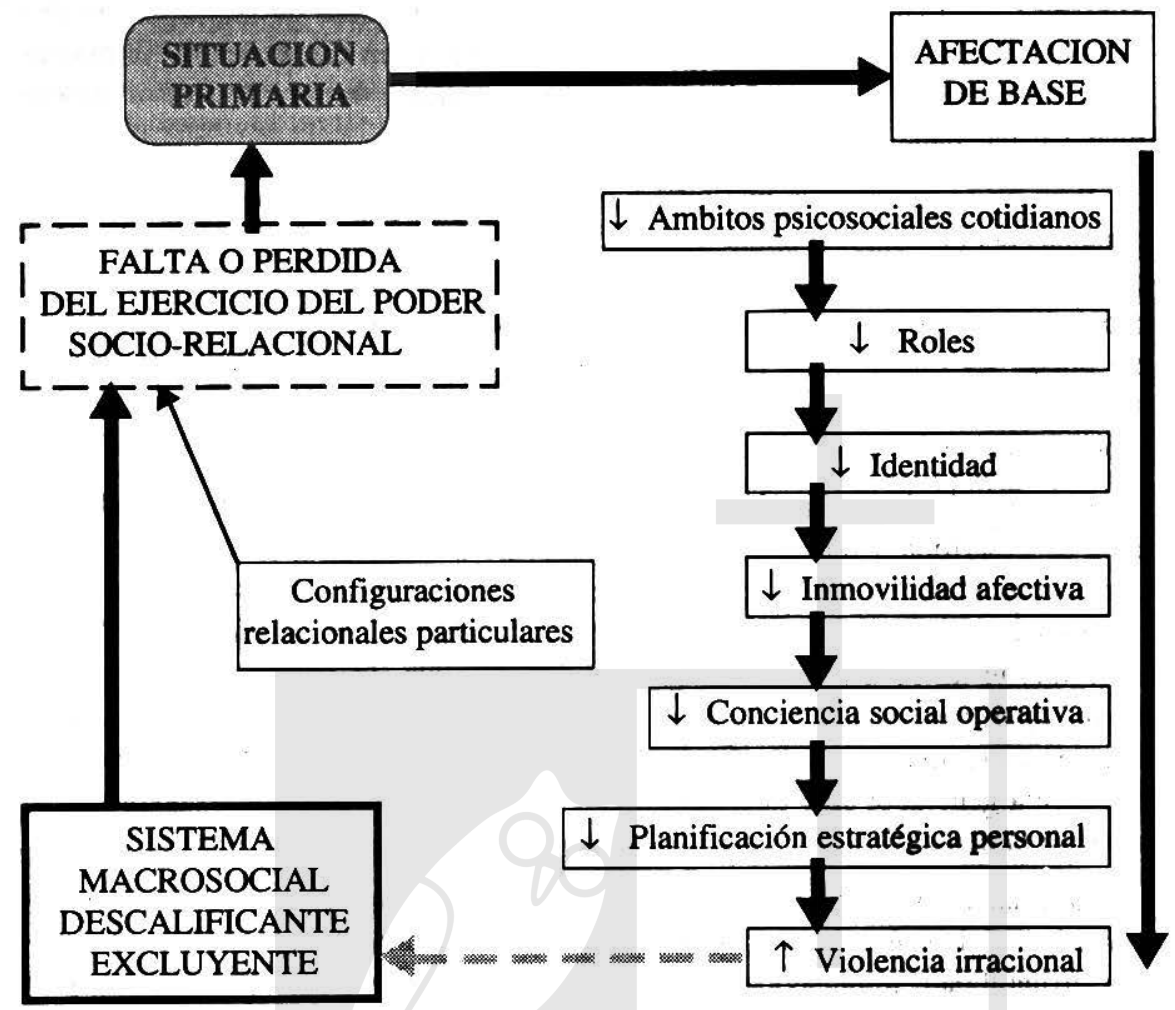

de cualquier guerra. Ellas no dependen de culturas de autoritarismo, a la inversa, son generadoras de esa cultura. No se trata de perderse en el "árbol" de la antropología o de la psicología, sino de profundizar, desde la perspectiva de la salud mental de base, en el conocimento del "bosque" del sistema que ha hecho orgánicas esas relaciones. Complementariamente, la búsqueda de precisión en la clínica de las afectaciones individuales, familiares, grupales, psicoambientales servirá para lograr mayor especificidad en los diagnósticos causales y más cercanía entre la práctica de cublculos y la intervención comunitaria.

Los sistemas macrosociales que funcionan con base en la descalificación "vertical" o en "cascada" de las personas, configuran instituciones que "succionan" el poder socio-relacional de las personas. Con ellos se aumenta el control del sistema sobre los individuos privados de ese poder. Entonces, se hace más fácil y "natural" someter las relaciones humanas a la ley del valor, que requiere establecer como norma universal la competencia excluyente y la generación permanente de mutilados psicosociales. Un sistema de esta naturaleza limita en los individuos su capaci- 
dad de reflejarse socialmente, con lo cual debilita su calidad y accionar como sujeto, y alimenta el potencial vulnerante de las dinámicas de poder institucional y de la infinidad de objetos materiales, de la cultura hegemónica, que se constituye en los principales vehículos para intemalizar ese sometimiento a su nivel subjetivo, volviendo más compleja y reactiva la afectación de salud mental de base.

San Salvador, febrero de 1998.

\section{Referenclas bibllogralticas}

Basaglia, F. et al. ¿Psiguiatria o ideologla de la locura?, Barcelona: Editoria] Anagrama, 1972.

Martín-Baro, I. Sistema, grupo y poder, San Salvador: UCA editores, 1993.

Sisti, E. Salud mental de base, San Salvador: UCA Editores, 1995.

Sisti, E. Psicoestinulacion de base, San Salvador: Editorial Universitaria, 1997.

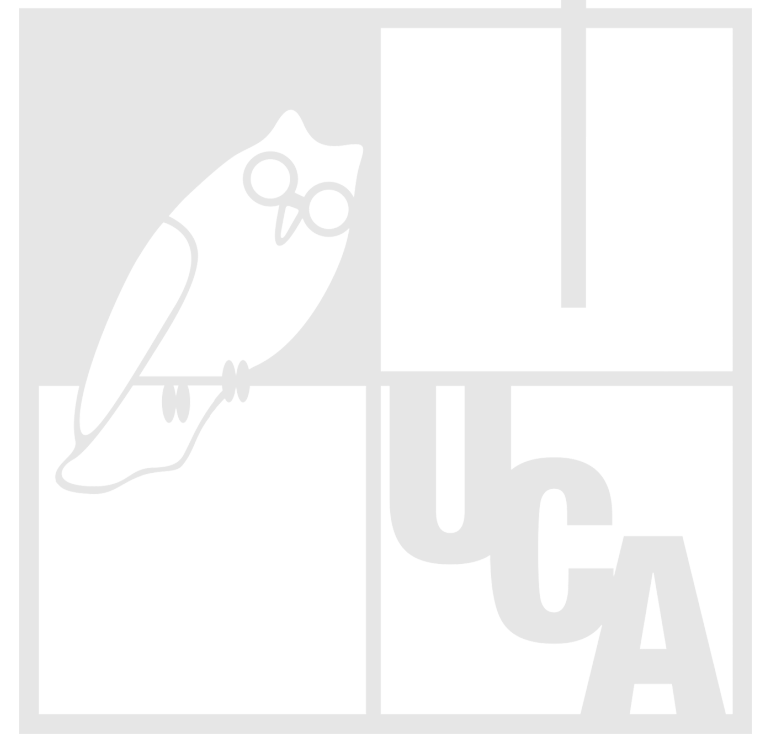

